TITLE:

\title{
Mode identifiability of a multi-span cable-stayed bridge utilizing stabilization diagram and singular values
}

\author{
$\operatorname{AUTHOR}(\mathrm{S}):$ \\ Goi, Y.; Kim, C.W.
}

\section{CITATION:}

Goi, Y.... [et al]. Mode identifiability of a multi-span cable-stayed bridge utilizing

stabilization diagram and singular values. Smart Structures and Systems 2016, 17(3): 391 411

ISSUE DATE:

2016-03-25

URL:

http://hdl.handle.net/2433/236011

RIGHT:

発行元の許可を得て掲載しています。This is not the published version. Please cite only the published version. この論文は出版社版でありませ ん。引用の際には出版社版をご確認ご利用ください。 


\title{
Mode Identifiability of a Multi-span Cable-stayed bridge Utilizing Stabilization Diagram and Singular Values
}

\author{
Y. Goi ${ }^{1}$ and C.W. Kim ${ }^{2}$ \\ ${ }^{1,2}$ Department of Civil and Earth Resources Engineering, Kyoto University, Kyoto 615-8540, Japan
}

(Received .....)

\begin{abstract}
This study investigates the mode identifiability of a multi-span cable-stayed bridge in terms of a benchmark study using stabilization diagrams of a system model identified using stochastic subspace identification (SSI). Cumulative contribution ratios (CCRs) estimated from singular values of system models under different wind conditions were also considered. Observations revealed that wind speed might influence the mode identifiability of a specific mode of a cable-stayed bridge. Moreover the cumulative contribution ratio showed that the time histories monitored during strong winds, such as those of a typhoon, can be modeled with less system order than under weak winds. The blind data Acc 1 and Acc 2 were categorized as data obtained under a typhoon. Blind data Acc 3 and Acc 4 were categorized as data obtained under wind conditions of critical wind speeds around $7.5 \mathrm{~m} / \mathrm{s}$. Finally, blind data Acc 5 and Acc 6 were categorized as data measured under weak wind conditions.
\end{abstract}

Keywords: benchmark study, cable-stayed bridge, cumulative contribution ratio, operational modal identification

\section{Introduction}

Managing aging civil infrastructure efficiently is a crucially important technical issue. Techniques of structural health monitoring (SHM) based on vibration measurements have been attracting bridge owners because of the increasing feasibility of efficient inspection. Today, modal properties of bridges such as natural frequencies, damping ratios and mode shape are identifiable from vibration data of bridges. However, changes in structural integrity engender changes in these modal properties of bridges (Zhang 2007, Kim et al. 2013). Consequently, vibration-based SHM is a useful technique if a bridge can be excited easily and effectively. Two commonly used means to excite bridges are forced vibration tests and ambient vibration tests. In forced vibration tests, bridges are excited by a shaker, impact hammer, or other means. However, ambient vibration tests use various natural excitations such as wind and ground motion. For bridge health monitoring, ambient vibration tests are regarded as being much more convenient than forced vibration tests because natural

\footnotetext{
1 Ph.D. student

${ }^{2}$ Corresponding author, Professor, E-mail: kim.chulwoo.5u@kyoto-u.ac.jp
} 
excitations require no traffic control, whereas the forced vibration tests require traffic control during testing.

Among outputs of various kinds, only operational modal identification techniques based on ambient vibrations have been proposed (e.g. Heylen et al. 1997) by assuming natural excitation as stationary white noise inputs. Such techniques have been applied to monitor real bridges (e.g. Peeters et al. 2006, Kim and Chang 2014a, Ni et al. 2015). Among many applications used for real bridge monitoring, Ni et al. (2015) reported that the modal shape of the second mode (deficient mode) of a cable-stayed bridge named Ting Kau Bridge is not identifiable under weak wind conditions, although it can be identified clearly under typhoon conditions. Prof. Y.Q. Ni of Hong Kong Polytechnic University has proposed a benchmark study to answer the following questions.

1) Why are the frequencies of deficient mode(s) identifiable, while modal shapes are unidentifiable?

2) Why do the modal shapes of deficient mode(s) not match each other well, in particular for modal components in a specific direction?

3) What is the damping performance of deficient modes under different excitations?

4) What are the modal force and modal energy performance under different excitations?

5) Does excitation (wind) direction also affect operational modal identifiability?

In a benchmark study, 13 samples of "non-blind" bridge vibration data with information about wind speed and 6 samples of "blind" data with no other information were offered. Investigations were conducted to assess the mode identifiability of operational modal analysis methods.

This study was conducted to investigate the mode identifiability of a cable-stayed bridge, particularly addressing its linear system model. We used a modal identification method called data-driven stochastic subspace identification (SSI) (Overschee and Moor 1996), and investigated mode identifiability, particularly addressing stabilization diagrams (Heylen et al. 1997, Chang et al. 2013) and cumulative contribution ratios (CCRs) (Kim et al. 2014b). The stabilization diagram is known as a diagram showing plots of estimated natural frequency with respect to the model order of the linear system model. A physically meaningful mode is believed to yield similar natural frequencies, although the model order is overspecified and therefore displays a nearly vertical line in the stabilization diagram: spurious modes might not. The SSI method requires singular value decomposition (SVD) in its computational processes. The use of CCRs enables evaluation of how much information is included in each singular value. This study adopts CCR to investigate the quantity of modal information included in the identified linear system model. 


\section{Investigated bridge and Benchmark data}

\subsection{Ting Kau Bridge and monitoring system}

Ting Kau Bridge (TKB) is a three-tower cable-stayed bridge with two main spans of 448 $\mathrm{m}$ and $475 \mathrm{~m}$, respectively, and two side spans of $127 \mathrm{~m}$ each as presented in Fig. 1 (Bergermann and Schlaich 1996). The bridge deck is separated respectively into two carriageways with a width of $18.8 \mathrm{~m}$ each. Between them are three slender single-leg towers with heights of $170 \mathrm{~m}, 194 \mathrm{~m}$, and $158 \mathrm{~m}$. Each carriageway comprises two longitudinal steel girders along the deck edges with steel cross girders at $4.5 \mathrm{~m}$ intervals, with a concrete slab on top. The two carriageways and their intervening $5.2 \mathrm{~m}$ gap are linked at $13.5 \mathrm{~m}$ intervals by connecting cross girders. The deck is supported by 384 stay cables in four cable planes.

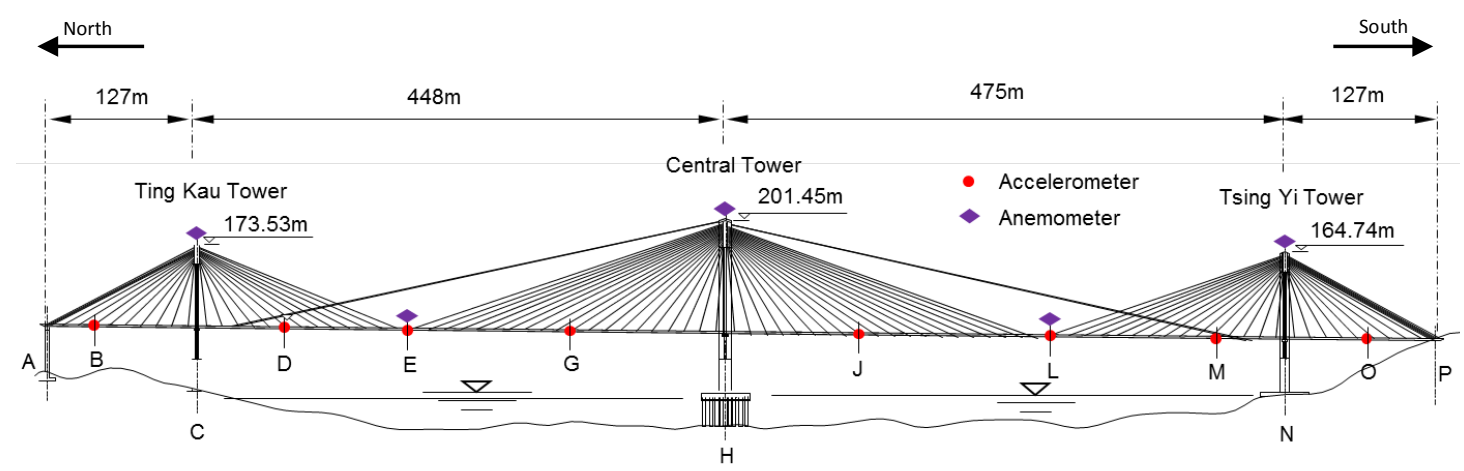

Fig. 1 Deployment of Accelerometers and Anemometers on Ting Kau Bridge.

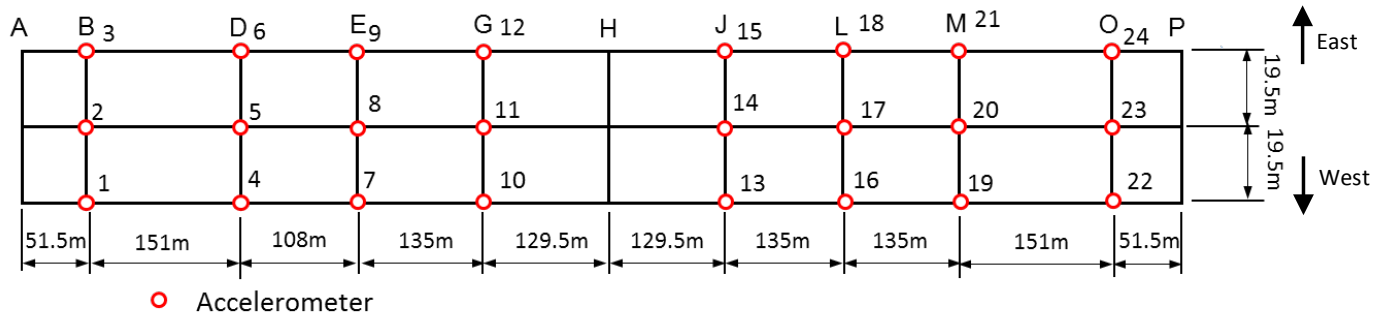

Fig. 2 Geometry configuration of accelerometers on the bridge deck.

As part of a long-term SHM system devised by the Hong Kong Special Administrative Region (SAR) Government Highways Department, more than 230 sensors were installed permanently on the TKB after completing bridge construction in 1999 (Wong 2004, Ko and Ni 2005).

The layout of the sensors at eight deck sections on the TKB is presented in Fig. 2. In each section, two accelerometers were installed on the east side and the west side of the longitudinal steel girders respectively to measure the vertical acceleration. One accelerometer 
was installed on the central cross girder to measure the transverse acceleration. The sampling frequency of accelerometers was $25.6 \mathrm{~Hz}$. Data acquired by the accelerometers deployed on the bridge deck are considered in the benchmark study.

\subsection{Benchmark data}

For the benchmark study, 13 samples of non-blind data and 6 samples of blind data are given. Table 1 presents the time duration and wind conditions of non-blind data. Data from Sample 1 to Sample 6 were measured under weak wind conditions. Data from Sample 7 to Sample 10 were measured under typhoon conditions. Data from Sample 11 to Sample 13 were measured at wind speeds around the critical value (threshold) of $7.5 \mathrm{~m} / \mathrm{s}$, at which the deficient mode becomes identifiable (Ni et al. 2015). Traffic was blocked during the typhoons.

Six given blind data samples designated as Acc 1, Acc 2, Acc 3, Acc 4, Acc 5, and Acc 6 are provided for the benchmark study. The blind dataset includes acceleration data obtained under weak wind conditions, typhoon conditions, and around the critical wind speed.

Table 1 Non-blind data

\begin{tabular}{|c|c|c|c|}
\hline Sample & Time duration & $\begin{array}{l}\text { Mean hourly wind speed } \\
\qquad(\mathrm{m} / \mathrm{s})\end{array}$ & Remarks \\
\hline Sample 1 & 15:00-16:00, 28 Dec. 1999 & 2.00 & \\
\hline Sample 2 & 15:00-16:00, 18 Feb. 1999 & 3.40 & \\
\hline Sample 3 & 15:00-16:00, 01 Mar. 1999 & 3.34 & \\
\hline Sample 4 & 15:00-16:00, 21 June 1999 & 3.41 & \\
\hline Sample 5 & 15:00-16:00, 24 July 1999 & 6.17 & \\
\hline Sample 6 & 15:00-16:00, 12 Aug. 1999 & 4.20 & \\
\hline Sample 7 & 03:00-04:00, 07 June 1999 & 12.11 & Typhoon: Maggie \\
\hline Sample 8 & 02:00-03:00, 23 Aug. 1999 & 15.62 & Typhoon: Sam \\
\hline Sample 9 & 06:00-07:00, 16 Sep. 1999 & 21.72 & Typhoon: York \\
\hline Sample 10 & 15:00-16:00, 16 Sep. 1999 & 15.91 & Typhoon: York \\
\hline Sample 11 & 08:00-09:00, 07 June 1999 & 7.36 & \\
\hline Sample 12 & 22:00-23:00, 16 Sep. 1999 & 7.77 & \\
\hline Sample 13 & 09:00-10:00, 26 Sep. 1999 & 7.43 & \\
\hline
\end{tabular}




\section{Mode Identification by Stochastic Subspace Identification}

\subsection{Stochastic Subspace Identification}

The dynamical system is modeled as the following state space model (Heylen et al. 1997, Overschee and Moor 1996).

$$
\begin{gathered}
\mathbf{x}(k+1)=\mathbf{A x}(k)+\mathbf{w}(k) \\
\mathbf{y}(k)=\mathbf{C x}(k)+\mathbf{v}(k)
\end{gathered}
$$

Therein, $\mathbf{x}(k)$ and $\mathbf{y}(k)$ respectively denote the state of structure and measurement at each time step $k$. Also, $\mathbf{w}(k)$ and $\mathbf{v}(k)$ respectively denote the process noise and measurement noise vectors. They are assumed to be stationary white noise. System matrices $\mathbf{A}$ and $\mathbf{C}$, which contain the modal information, are estimated using least-squares method for the minimal prediction error of state $\mathbf{x}(k)$ given by the forward Kalman filter. The poles of the dynamical system provide modal properties of the dynamical system.

The algorithm for the SSI is described briefly. First, we obtain the oblique projection matrix $\boldsymbol{O}_{i}$, as estimated from Eq. (2).

$$
\boldsymbol{O}_{i}=\mathbf{Y}_{\mathrm{f}} \mathbf{Y}_{\mathrm{p}}^{\mathrm{T}}\left(\mathbf{Y}_{\mathrm{p}} \mathbf{Y}_{\mathrm{p}}^{\mathrm{T}}\right)^{\dagger} \mathbf{Y}_{\mathrm{p}}
$$

Therein, $(\cdot)^{\dagger}$ denotes the Moore-Penrose pseudo-inverse matrix. $\mathbf{Y}_{\mathrm{f}}$ and $\mathbf{Y}_{\mathrm{p}}$ are defined as follows, respectively representing the block Hankel matrices of the future and past outputs.

$$
\begin{gathered}
\mathbf{Y}_{\mathbf{p}}=\left[\begin{array}{cccc}
\mathbf{y}(0) & \mathbf{y}(1) & \ldots & \mathbf{y}(j-1) \\
\ldots & \ldots & \ldots & \ldots \\
\mathbf{y}(i-2) & \mathbf{y}(i-1) & \ldots & \mathbf{y}(i+j-3) \\
\mathbf{y}(i-1) & \mathbf{y}(i) & \ldots & \mathbf{y}(i+j-2)
\end{array}\right] \\
\mathbf{Y}_{\mathbf{f}}=\left[\begin{array}{cccc}
\mathbf{y}(i) & \mathbf{y}(i+1) & \ldots & \mathbf{y}(i+j-1) \\
\ldots & \ldots & \ldots & \ldots \\
\mathbf{y}(2 i-2) & \mathbf{y}(2 i-1) & \ldots & \mathbf{y}(2 i+j-3) \\
\mathbf{y}(2 i-1) & \mathbf{y}(2 i) & \ldots & \mathbf{y}(2 i+j-2)
\end{array}\right]
\end{gathered}
$$

The singular value decomposition (SVD) is then applied to factorize $\boldsymbol{O}_{i}$ as shown below.

$$
\boldsymbol{O}_{i}=\mathbf{U S V}^{\mathbf{T}}=\left(\mathbf{U}_{\mathbf{1}} \mathbf{U}_{\mathbf{2}}\right)\left(\begin{array}{cc}
\mathbf{S}_{\mathbf{1}} & \mathbf{0} \\
\mathbf{0} & \mathbf{S}_{\mathbf{2}}
\end{array}\right)\left(\mathbf{V}_{\mathbf{1}} \mathbf{V}_{\mathbf{2}}\right)^{\mathbf{T}} \simeq \mathbf{U}_{\mathbf{1}} \mathbf{S}_{\mathbf{1}} \mathbf{V}_{\mathbf{1}}^{\mathbf{T}}
$$

In those equations, $\mathbf{U}$ and $\mathbf{V}$ are unitary matrices with an appropriate size; $\mathbf{S}$ is a diagonal matrix with non-negative elements. Diagonal elements of $\mathbf{S}$ are known as singular values of $\boldsymbol{O}_{i}$. Singular values in $\mathbf{S}$ are listed in descending order. Therefore, the components in $\mathbf{U}_{\mathbf{1}} \mathbf{S}_{\mathbf{1}} \mathbf{V}_{\mathbf{1}}^{\mathbf{T}}$ include most of the information defining the elements in $\boldsymbol{O}_{i}$. Components in $\mathbf{U}_{2} \mathbf{S}_{2} \mathbf{V}_{2}^{\mathbf{T}}$ are regarded as trivial components. Theoretically, the optimal state sequence $\mathbf{X}_{i}=\left[\begin{array}{llll}\mathbf{x}(i) & \mathbf{x}(i+1) & \ldots & \mathbf{x}(i+j-1)\end{array}\right]$ predicted by the Kalman filter in a least squares sense is obtained as shown below. 


$$
X_{i}=S_{1}^{1 / 2} V_{1}^{T}
$$

The significant components of orthogonal vectors in the state sequence can be extracted by application of SVD to $\boldsymbol{O}_{i}$. The system matrices are obtainable from $\mathbf{X}_{i}$. The number of poles corresponds to the number of singular values determined in Eq. (4). In other words, we can extract the significant modal components of the bridge from the measured acceleration data by the SVD.

\subsection{Procedure for mode identification}

To discuss the mode identifiability, this study uses the stabilization diagram to determine the stable modes. The procedure for the mode identification adopted in this study is depicted in Fig. 3, in which the maximum model order $n=80$ was decided to be the cumulative contribution ratio of singular values reaching $90 \%$, even in a noisy system, as discussed in Section 4.2. The frequency range of interest of TKB is below $0.5 \mathrm{~Hz}$. A concern related to modal identification below $0.5 \mathrm{~Hz}$ is that higher-frequency information interferes with it. This study adopted a decimation filter to avoid the effects of high-frequency vibration. The lowpass Chebyshev type1 IIR filter of order 8 (Daniels 1974) was used.

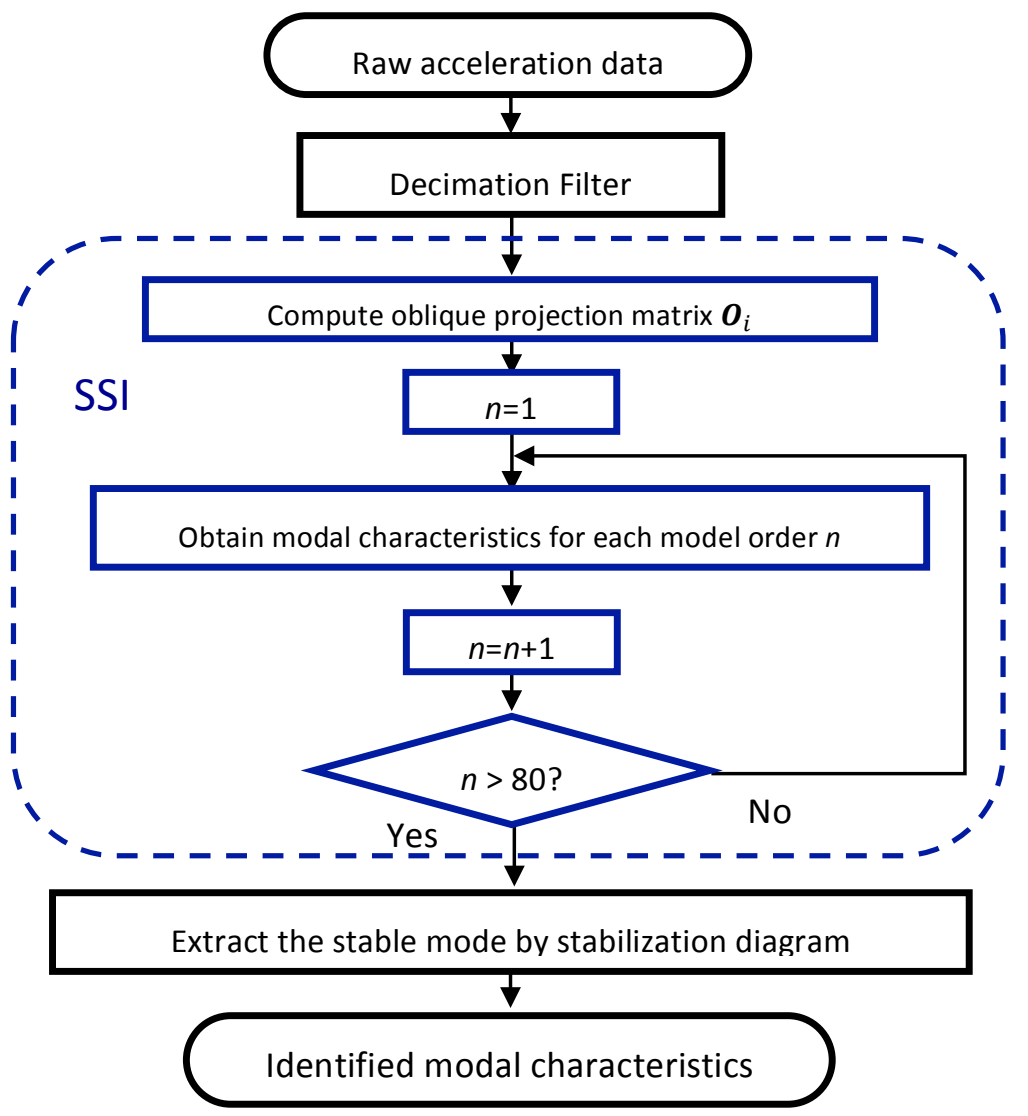

Fig. 3 Modal identification procedure using SSI. 
Modal characteristics of each model order were identified considering the same size of the block Hankel matrix of measurement data, i.e., $i$ in Eq. (3). For quantitative evaluation of the optimal size of the block Hankel matrix, this study considered the Akaike information criterion (AIC) obtained from the prediction error of estimated system models calculated from each model order without being factorized by SVD. Theoretically, the minimum AIC is known to produce the best model for estimating actual phenomena (Akaike 1973).

The AICs of each system model estimated from the non-blind data of Sample 1 to Sample 13 are presented in Fig. 4 with respect to the block Hankel matrix size, in which the optimal model order providing the best estimation of each measurement is marked with a circle. No clear correlation between AIC and wind condition was found from Fig. 4. It is noteworthy that $i=9$, which is the mean value of the optimal size of the block Hankel matrix of 13 non-blind data, was obtained from Fig. 4. It is used throughout this study.

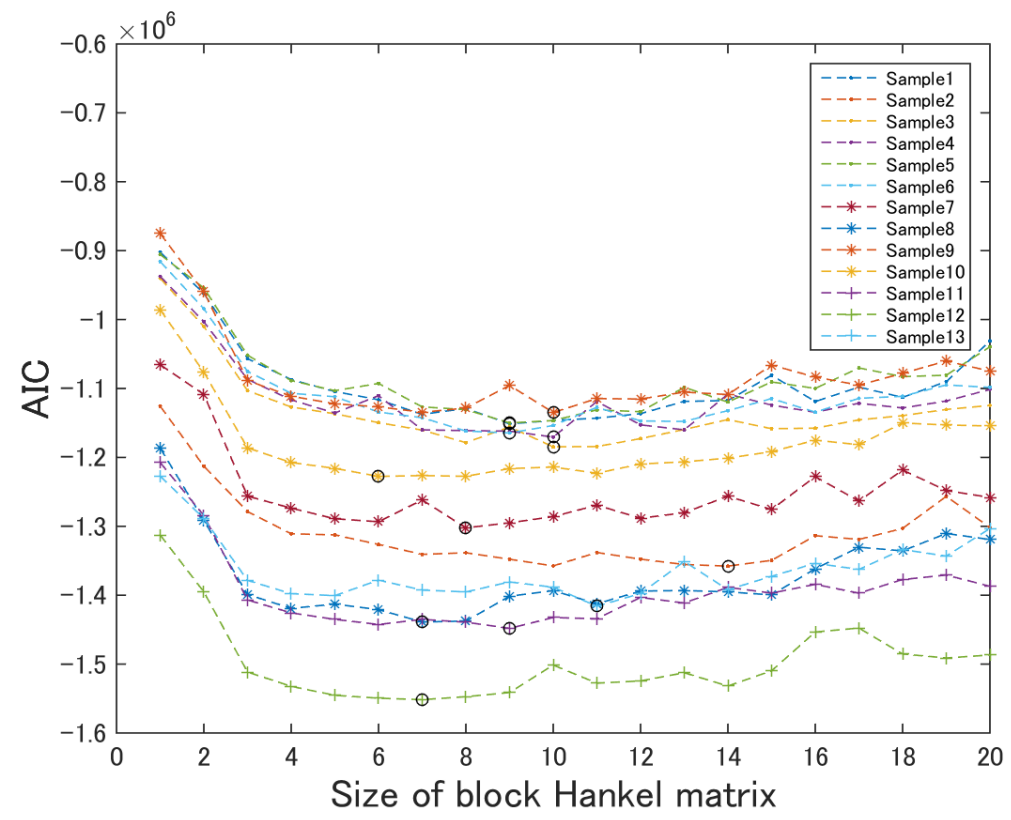

Fig. 4 AIC obtained from identified model by SSI.

This study considered the following stability criteria to extract stable modes.

$$
\begin{gathered}
\text { for } f_{p}: f_{p+\kappa}-f_{\epsilon}<f_{p}<f_{p+\kappa}+f_{\epsilon} \\
\text { for } \zeta_{p}: \zeta_{p+\kappa}-\zeta_{\epsilon}<\zeta_{p}<\zeta_{p+\kappa}+\zeta_{\epsilon} \\
\quad \text { for } \psi_{p}: \operatorname{MAC}_{l}<\operatorname{MAC}_{(p, p+\kappa)} \\
\quad\left(\kappa=-\kappa_{\epsilon}, \ldots,-1,1, \ldots, \kappa_{\epsilon}\right)
\end{gathered}
$$

In those expressions, $f_{p}, \zeta_{p}, \psi_{p}$ respectively denote the natural frequency, damping ratio, and modal shape with model order $p . \mathrm{MAC}_{(p, p+\kappa)}$ stands for the modal assurance criterion (MAC) value between modal shapes $\psi_{p}$ and $\psi_{p+\kappa}$. The MAC value is estimated by Eq. (7). 


$$
\operatorname{MAC}_{(p, p+\kappa)}=\frac{\left\|\psi_{p}^{T} \psi_{p+\kappa}\right\|^{2}}{\left\|\psi_{p}\right\|^{2}\left\|\psi_{p+\kappa}\right\|^{2}}
$$

Parameters $f_{\epsilon}, \zeta_{\epsilon}, \mathrm{MAC}_{l}$ in Eq. (6) are thresholds for the tolerable range of variation of each modal property; $\kappa_{\epsilon}$ is the range of model order that evaluates the inequalities.

This study adopted a strict rule to extract stable modes: stable modes must satisfy all the criteria in Eq. (6). The adopted thresholds were $f_{\epsilon}=0.005 \mathrm{~Hz}, \zeta_{\epsilon}=0.5 \%, \mathrm{MAC}_{l}=0.95$, and $\kappa_{\epsilon}=3$ in the frequency band lower than $0.5 \mathrm{~Hz}$. Poles with a negative damping ratio were ignored.

\section{Mode Identifiability of Non-Blind Data}

\subsection{Identified modal properties}

Natural frequencies, damping ratios, and mode shapes of each non-blind data were identified following the procedure described in Section 3.2. Identified natural frequencies are presented in Table 2. The identified damping ratios are presented in Table 3. A noteworthy point is that the modes with blanks in Tables 2 and 3 show unstable modes. That is, the poles of these modes are too unstable to satisfy the stability criteria. Therefore, they are expelled from the identification. The identified results from non-blind data are comparable to results reported in a previous study (Ni et al. 2015), i.e. the second mode $(0.23 \mathrm{~Hz})$ is not identified under weak wind conditions. The third mode for Sample 11 and fifth modes for Sample 1, Samples 3-5, and Sample 13 were not identified.

Table 2 Identified natural frequencies $(\mathrm{Hz})$

\begin{tabular}{|c|c|c|c|c|c|c|c|c|c|c|c|c|c|}
\hline \multirow{3}{*}{$\begin{array}{c}\text { Mode } \\
\text { No. }\end{array}$} & \multicolumn{13}{|c|}{ Natural Frequencies (Hz) } \\
\hline & \multicolumn{6}{|c|}{ Weak Wind } & \multicolumn{4}{|c|}{ Typhoon } & \multicolumn{3}{|c|}{ Critical Wind } \\
\hline & S1 & $\mathbf{S 2}$ & $\mathbf{S 3}$ & S4 & S5 & S6 & S7 & S8 & S9 & S10 & S11 & S12 & S13 \\
\hline 1 & 0.161 & 0.161 & 0.162 & 0.163 & 0.162 & 0.164 & 0.167 & 0.164 & 0.165 & 0.165 & 0.168 & 0.165 & 0.168 \\
\hline 2 & - & - & - & - & - & - & 0.227 & 0.227 & 0.227 & 0.226 & 0.229 & 0.228 & 0.227 \\
\hline 3 & 0.255 & 0.255 & 0.258 & 0.257 & 0.258 & 0.260 & 0.263 & 0.264 & 0.257 & 0.260 & - & 0.264 & 0.265 \\
\hline 4 & 0.287 & 0.287 & 0.284 & 0.286 & 0.282 & 0.285 & 0.289 & 0.292 & 0.287 & 0.289 & 0.282 & 0.290 & 0.291 \\
\hline 5 & - & 0.294 & - & - & - & 0.293 & 0.297 & 0.301 & 0.300 & 0.300 & 0.298 & 0.300 & - \\
\hline 6 & 0.307 & 0.310 & 0.308 & 0.315 & 0.312 & 0.318 & 0.323 & 0.324 & 0.319 & 0.317 & 0.315 & 0.322 & 0.323 \\
\hline 7 & 0.352 & 0.359 & 0.354 & 0.354 & 0.348 & 0.353 & 0.357 & 0.361 & 0.346 & 0.350 & 0.329 & 0.355 & 0.361 \\
\hline 8 & 0.358 & 0.364 & 0.360 & 0.358 & 0.357 & 0.358 & 0.361 & 0.368 & 0.358 & 0.359 & 0.361 & 0.362 & 0.367 \\
\hline 9 & 0.373 & 0.374 & 0.374 & 0.372 & 0.372 & 0.374 & 0.374 & 0.373 & 0.374 & 0.372 & 0.374 & 0.379 & 0.377 \\
\hline
\end{tabular}

*S1, Sample 1 ... S13, Sample 13 
Table 3 Identified damping ratios

\begin{tabular}{|c|c|c|c|c|c|c|c|c|c|c|c|c|c|}
\hline \multirow{3}{*}{$\begin{array}{c}\text { Mode } \\
\text { No. }\end{array}$} & \multicolumn{13}{|c|}{ Damping Ratios (\%) } \\
\hline & \multicolumn{6}{|c|}{ Weak Wind } & \multicolumn{4}{|c|}{ Typhoon } & \multicolumn{3}{|c|}{ Critical Wind } \\
\hline & S1 & $\mathbf{S 2}$ & S3 & S4 & S5 & S6 & S7 & S8 & S9 & S10 & S11 & S12 & S13 \\
\hline 1 & 0.82 & 0.69 & 1.01 & 0.93 & 0.95 & 1.10 & 1.57 & 1.72 & 3.38 & 1.54 & 1.31 & 1.07 & 1.11 \\
\hline 2 & - & - & - & - & - & - & 0.81 & 0.51 & 0.62 & 0.71 & 1.14 & 1.32 & 0.78 \\
\hline 3 & 1.75 & 1.83 & 1.43 & 1.37 & 3.76 & 1.30 & 0.82 & 0.74 & 3.12 & 1.39 & - & 0.69 & 0.61 \\
\hline 4 & 1.95 & 1.76 & 2.85 & 1.94 & 3.15 & 4.14 & 1.19 & 1.47 & 1.35 & 1.59 & 1.11 & 2.00 & 0.49 \\
\hline 5 & - & 0.67 & - & - & - & 1.95 & 1.68 & 1.08 & 0.90 & 1.58 & 1.52 & 0.61 & - \\
\hline 6 & 2.34 & 2.36 & 3.26 & 2.30 & 2.62 & 2.08 & 0.91 & 0.87 & 1.18 & 0.89 & 0.86 & 0.84 & 0.49 \\
\hline 7 & 1.57 & 0.67 & 1.68 & 2.03 & 3.76 & 5.69 & 3.49 & 1.09 & 2.79 & 2.89 & 1.27 & 2.19 & 1.09 \\
\hline 8 & 0.82 & 1.21 & 0.95 & 0.96 & 0.77 & 0.81 & 1.20 & 2.51 & 1.04 & 0.91 & 0.49 & 0.88 & 1.43 \\
\hline 9 & 1.16 & 1.52 & 1.25 & 0.99 & 1.34 & 1.13 & 1.90 & 0.87 & 1.43 & 0.84 & 0.67 & 1.79 & 1.98 \\
\hline
\end{tabular}

*S1, Sample 1 ... S13, Sample 13

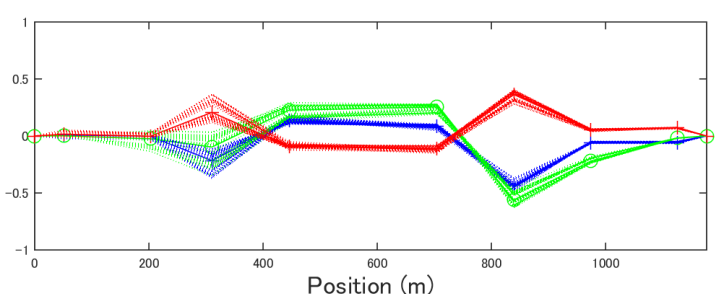

a) Sample 7

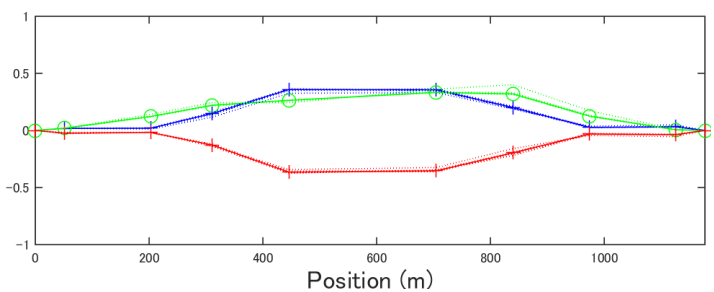

c) Sample 9

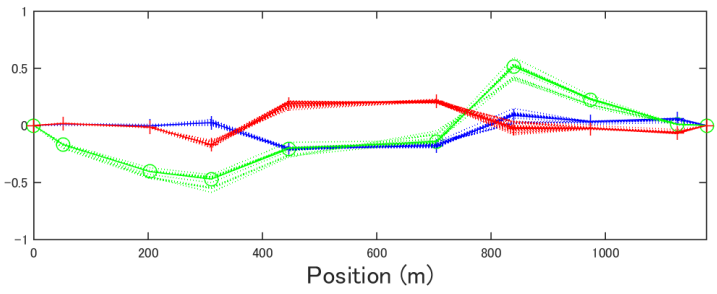

e) Sample 11

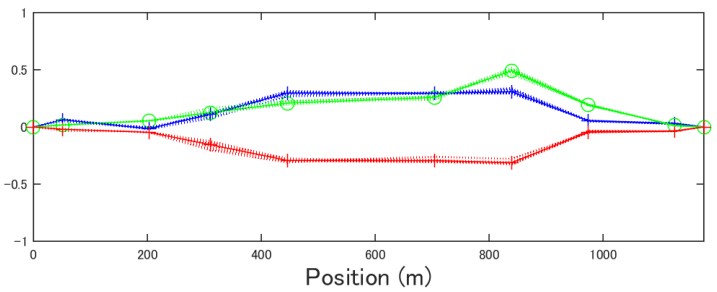

g) Sample 13

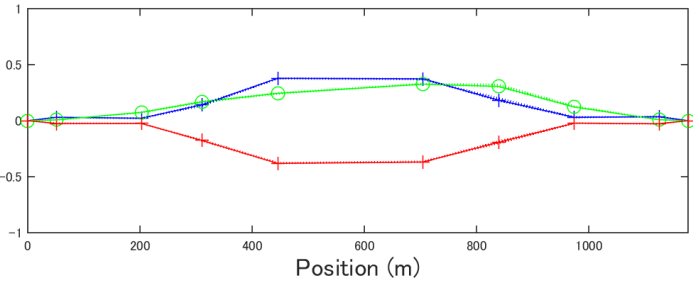

b) Sample 8

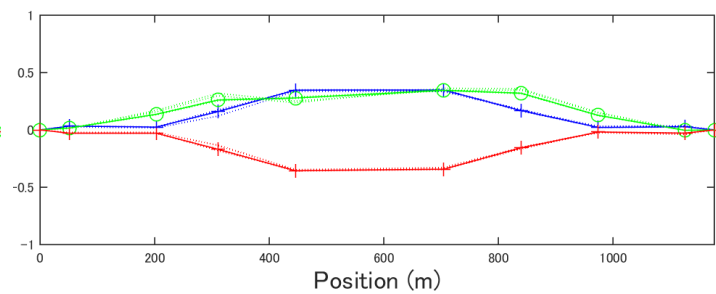

d) Sample 10

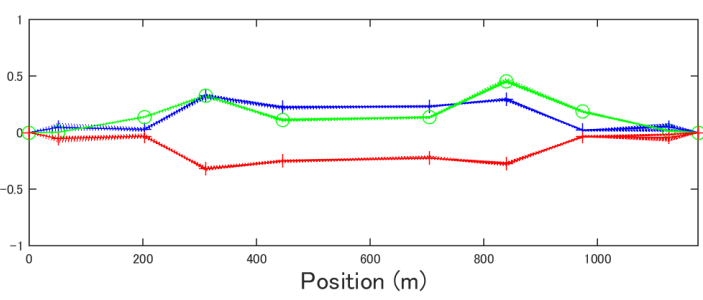

f) Sample 12

Fig. 5 Identified second mode shapes under typhoon and critical wind conditions. 
Smart Structures and Systems, An Int J., Vol.17, No. 3, pp.391-411, 2016.

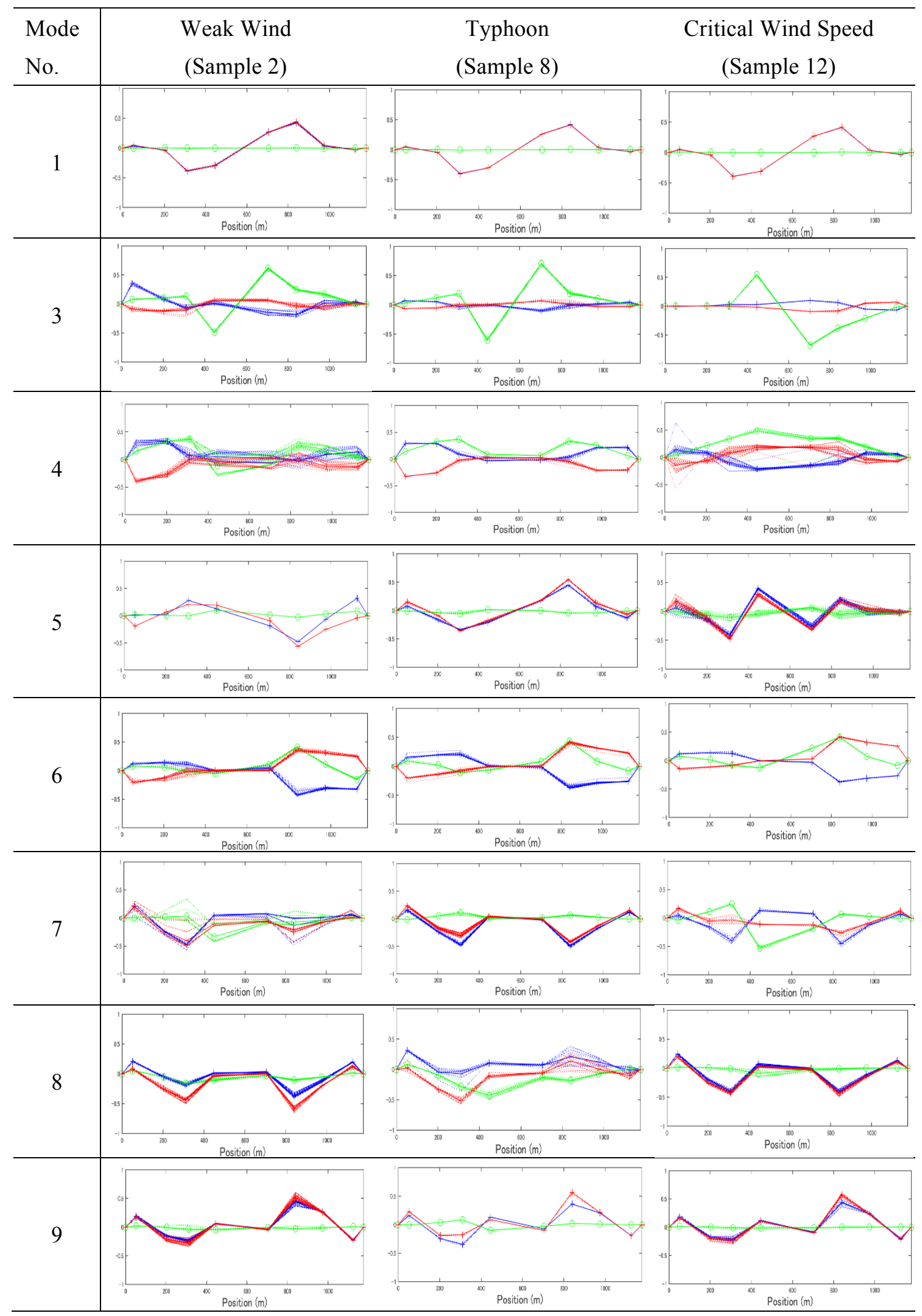

Fig. 6 Examples of the identified mode shapes except the second mode. 
Identified second mode shapes from Sample 7 to Sample 13 are presented in Fig. 5. The dotted lines show the identified modal shapes obtained by Eq. (6). The solid lines show their mean values. Lines with cross marks and circle marks respectively show the vertical mode shapes and lateral mode shapes. Under typhoon conditions, the second mode shape looks mutually comparable with others, except for Sample 7. The second mode shape from Sample 7 seems vague compared with other samples under typhoon conditions, even though the previous study (Ni et al. 2015) reported that the second mode shape obtained from Sample 7 was identical to those of the other samples. This point will be discussed later considering the unsteadiness of the excitation. The second mode shapes from critical wind conditions differed from the mode shapes identified from Samples 8-10. However, the second mode shapes identified from Samples 12 and 13 were comparable with the second mode shapes from Samples 8-10.

Mode shapes obtained from Sample 2 (weak wind condition), Sample 8 (typhoon condition), and Sample 12 (critical condition) are presented in Fig. 6. The fourth and fifth modes are not mutually comparable. The seventh mode shape identified from Sample 8 is more comparable with eighth mode shapes of Sample 2 and Sample 12 rather than their seventh mode shapes. Table 2 shows that this point implies difficulty in distinguishing these modes by merely depending on their natural frequencies because their natural frequencies are very similar. Natural frequencies and the first, third, sixth, and ninth mode shapes were identifiable with less variation, except for deficient modes. However, for identified damping ratios, greater variation than frequencies was observed as shown in Table 3.

\subsection{Mode identifiability}

Stabilization diagrams of non-blind data are also examined to find possible answers to the questions of the benchmark study. Figure 7, Fig. 8, and Fig. 9 respectively portray stabilization diagrams of data obtained under weak wind, typhoon, and critical wind conditions. The model order is considered from 1 to 80 for each figure.

The stabilization diagrams portrayed in Fig. 7 demonstrate that the second mode $(0.23 \mathrm{~Hz})$ was not identified under weak wind conditions. The diagrams show that unstable poles are scattered widely in the frequency band below $0.23 \mathrm{~Hz}$. No clear stable poles were observed on the frequency of the second mode $(0.23 \mathrm{~Hz})$. However, under typhoon conditions, fewer unstable poles were observed in the frequency band below $0.23 \mathrm{~Hz}$ as presented in Fig. 8. In other words, the typhoon condition led to stable poles related to the second mode. Regarding the stabilization diagram showing the critical wind condition, Fig. 9 shows that the distribution of the unstable poles below $0.23 \mathrm{~Hz}$ of Sample 11 and Sample 12 were similar to stabilization diagrams identified from typhoon conditions. In the stabilization diagram of 
Sample 13, many more unstable poles were identified than for Sample 11 and Sample 12, which implies that those unstable poles do not merely depend on the mean hourly wind speed.

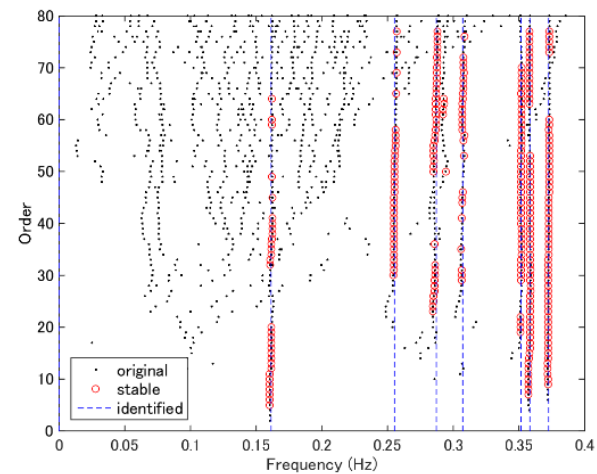

a) Sample 1

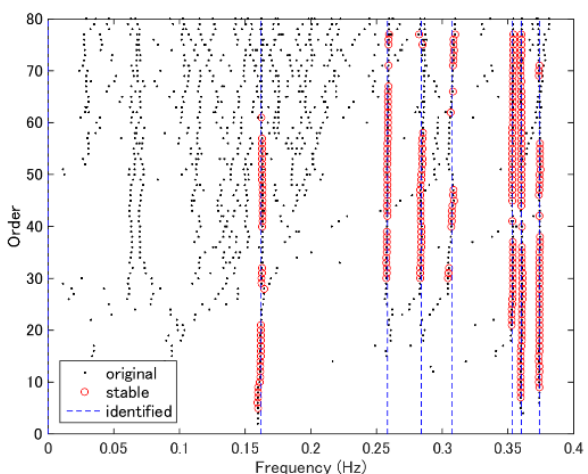

c) Sample 3

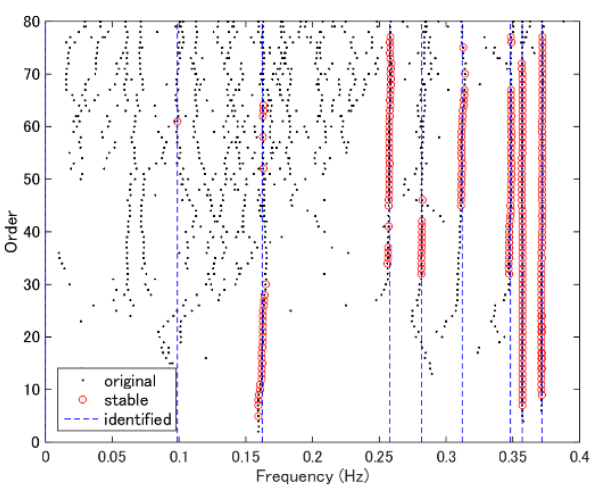

e) Sample 5

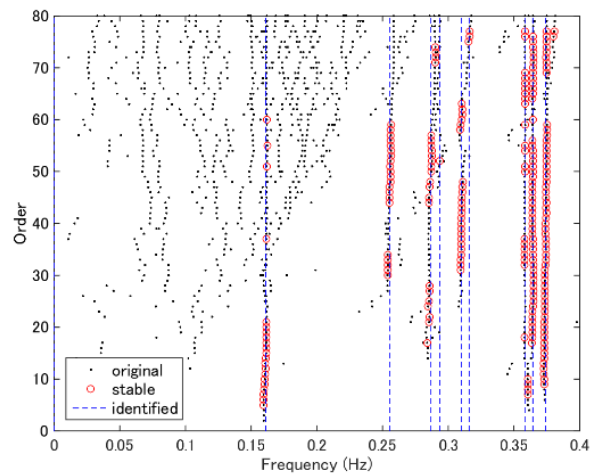

b) Sample 2

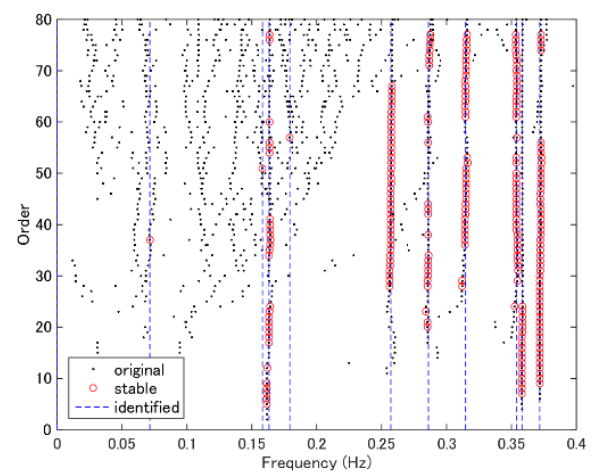

d) Sample 4

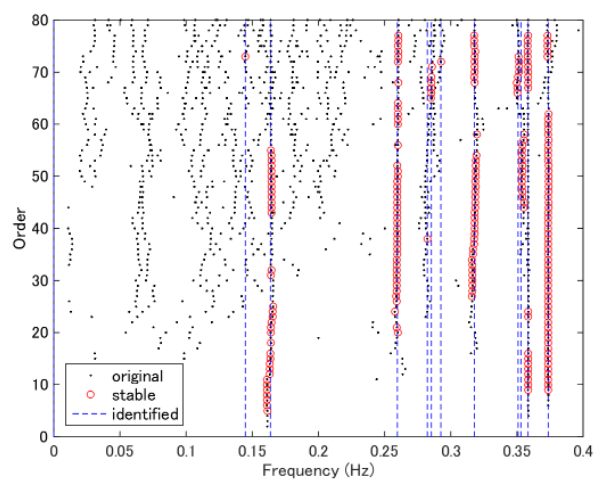

f) Sample 6

Fig. 7 Stabilization diagrams under the weak wind conditions. 


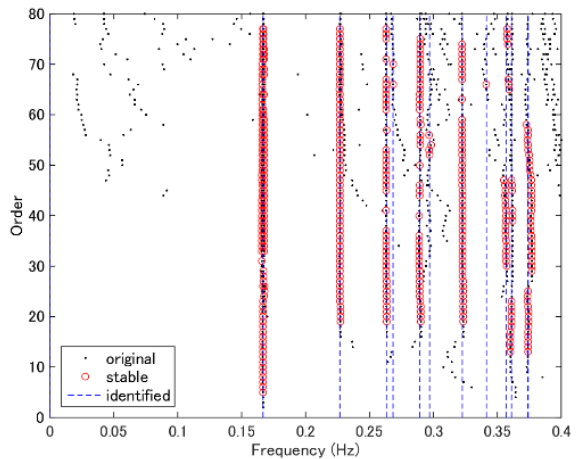

a) Sample 7

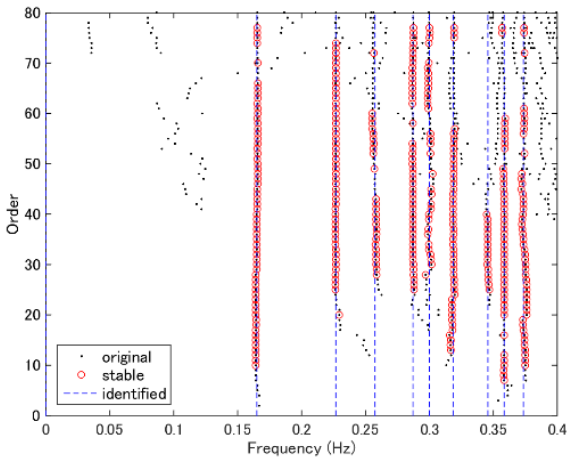

c) Sample 9

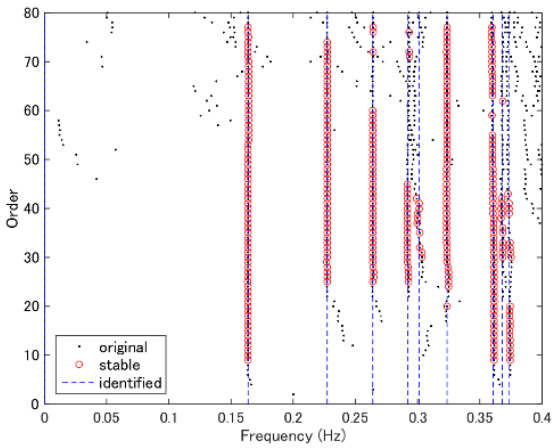

b) Sample 8

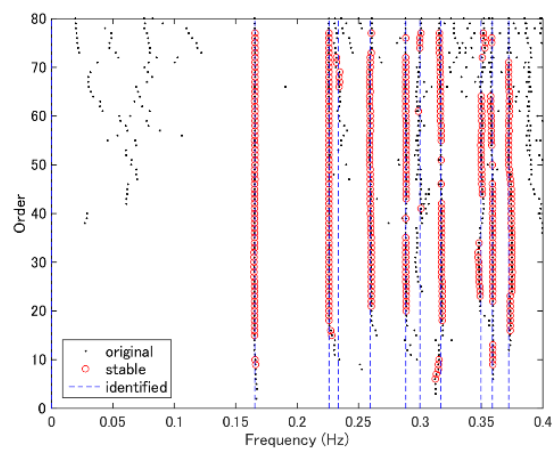

d) Sample 10

Fig. 8 Stabilization diagrams under the typhoon conditions.

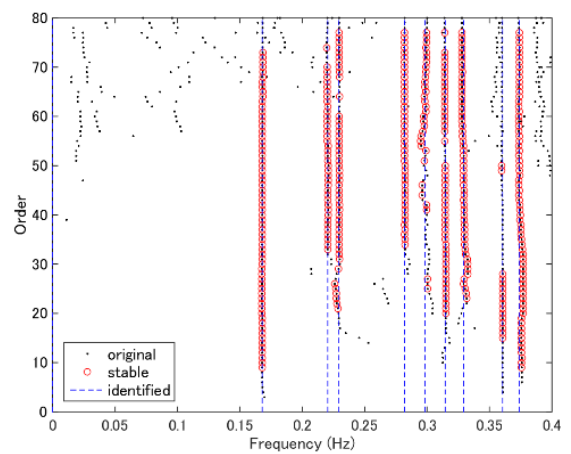

a) Sample 11

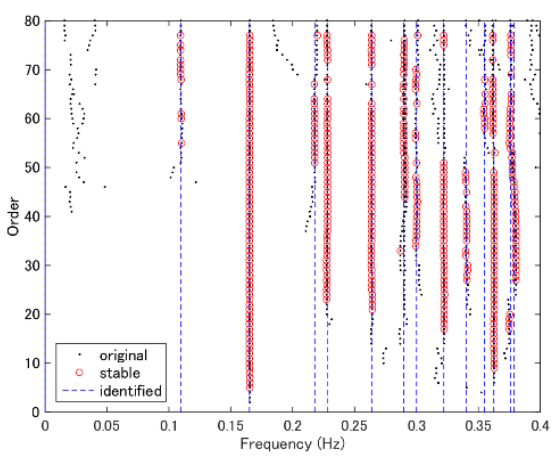

b) Sample 12

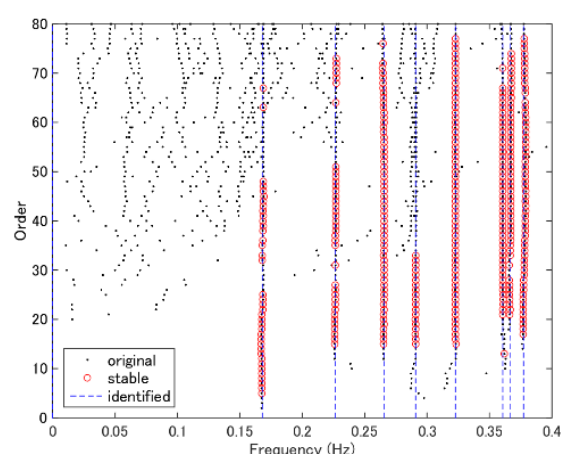

c) Sample 13

Fig. 9 Stabilization diagrams under the critical conditions. 
The observation described above from the stabilization diagrams in Figs. 7 and 8 demonstrated that lower modes were contaminated easily by unknown noise under weak wind conditions. However, under typhoon conditions, structural modes were well excited. The system matrix is expected to be dominated by structural information rather than noise, which might link with mode identifiability of the second mode. This point will be discussed later using power spectral density (PSD) curves and the CCR from singular values of the system.

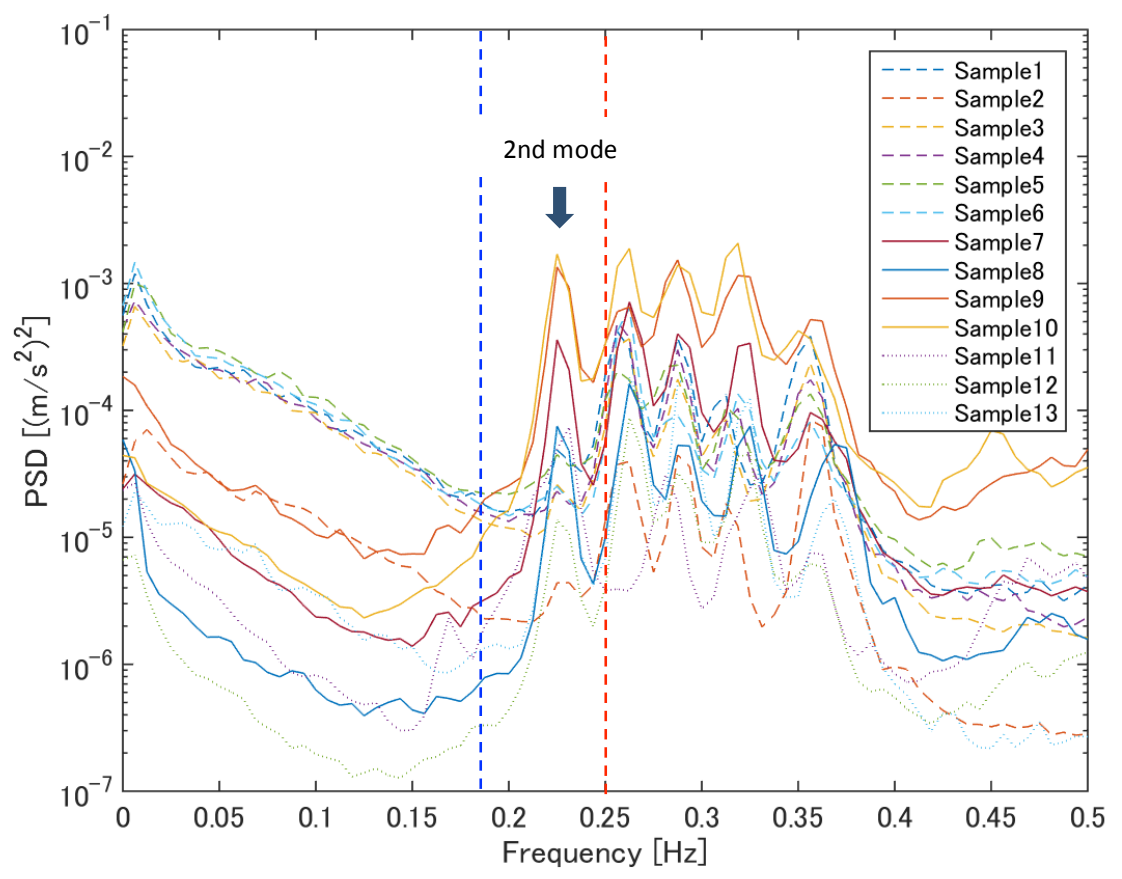

Fig. 10 Mean value of the lateral acceleration PSD.

The mean values of the PSD of lateral accelerations for each sample are presented in Fig. 10, which shows that the bridge vibrations of the frequency band below $0.23 \mathrm{~Hz}$ in the transverse direction under weak wind conditions showed higher PSD than those under typhoon or critical conditions. Especially for the low frequency band below $0.18 \mathrm{~Hz}$, most PSDs of the transverse accelerations under the weak winds exceed the PSDs of the typhoon and critical conditions, even though the exciting wind speed is less than that in typhoon conditions. A possible reason might be that the influence of traffic was controlled during the typhoon.

To investigate differences of identified systems under different wind conditions, the CCR of singular values of the system is considered. The CCR of model order $n$ is defined as shown in Eq. (8).

$$
C C R=\frac{\sum_{i=1}^{n} s_{i}}{\sum_{i=1}^{N} s_{i}}
$$


Therein, $s_{i}$ is the $i$-th singular value in Eq. (4). $N$ is the total number of the singular values.

The CCR estimated from all the non-blind data is shown with respect to the model order as presented in Fig. 11. Results show that, in considering the same model order, the typhoon condition caused higher CCR than the weak wind condition did. In other words, in considering same model order, the CCR under the typhoon condition includes more information about the system than that under the weak wind condition. Therefore, vibrations of the bridge under typhoon conditions can be modeled as a dynamic system with lower model order. For example, a system model containing $90 \%$ of the measurement information can be described by the model order of 50 under a typhoon condition (see the vertical broken lines at the model order of 50 in Fig. 11). However, a model order of 80 is necessary to include $90 \%$ of the system information under weak wind conditions (see vertical broken lines near the model order of 90 in Fig. 11). The CCRs obtained from Sample 11 and Sample 12 showed similar trends with CCRs of the typhoon condition. The CCR of Sample 13 took $90 \%$ of the system information with model order of 80 , like those under the weak wind condition. However, the CCR of Sample 13 included similar amounts of system information such as the typhoon condition under the lower model order of 15 . The observation described above implies that the spurious poles observed in Figs. 7, 8, and 9 reduced the quantity of information included in the identified system models.

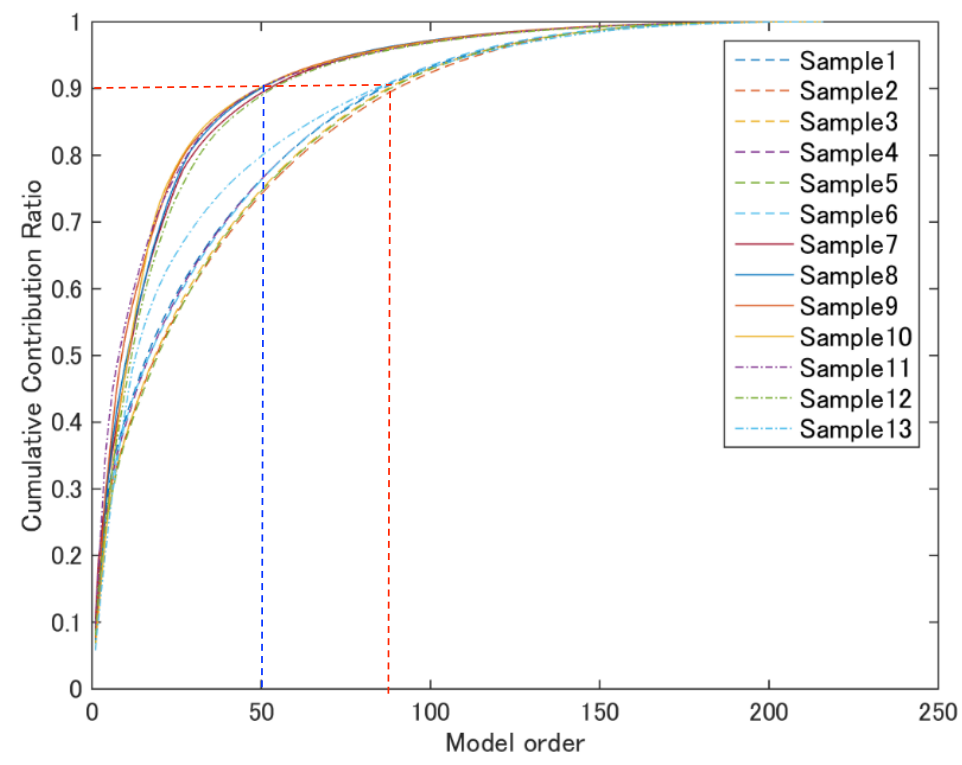

Fig. 11 Cumulative contribution ratios of singular values calculated in SSI.

As described previously, the second mode shape under the typhoon condition (Sample 7) was not comparable with other typhoon condition, as presented in Fig. 5, even though the report described that the mode is clearly identifiable. We therefore investigated the time 
history of the root mean square (RMS) of measured vertical acceleration of Sample 7 as Fig. 12 to ascertain any possible reason. This figure shows that the amplitude of the vertical acceleration tends to be larger in the last several minutes. That observation implies that the excitation affecting the bridge is not steady in the last several minutes. This unsteadiness of the excitation can be one cause of uncertainty of the modal parameters because SSI method is based on the state space model described as Eq. (1), which assumes the excitation as steady white noise. Figure 13 shows the second mode shape identified from the first $50 \mathrm{~min}$ of the measurement data of Sample 7: the former part of Fig. 12 separated by the broken line. This figure shows the second mode as clearly identifiable as the other samples under typhoon conditions. This result implies that the unsteadiness of the excitation can be one cause of a failure to identify the mode shapes.

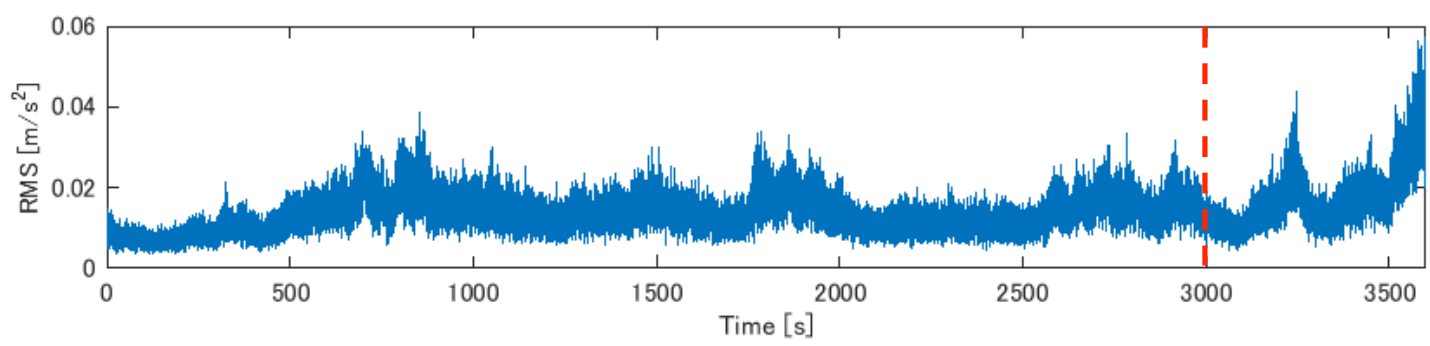

Fig. 12 Time history of the RMS of measured vertical acceleration of Sample 7.

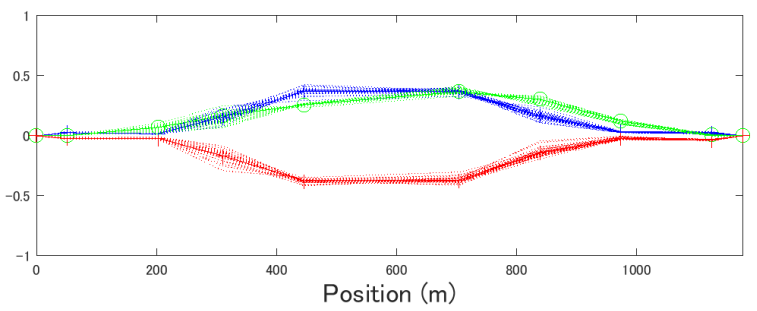

Fig. 13 Second mode shape identified from the first $50 \mathrm{~min}$ of the data of Sample 7.

\section{Mode Identifiability of Blind Data}

Blind data were designated as Acc 1, Acc 2, Acc 3, Acc 4, Acc 5, and Acc 6 given for the benchmark study. The SSI, stabilization diagram and the CCR are also used to identify modal properties and to examine the mode identifiability of the blind data. The blind dataset includes two sets of data obtained under weak wind excitation, two sets under typhoon excitation, and two sets under wind speeds of around $7.5 \mathrm{~m} / \mathrm{s}$.

Stabilization diagrams of the blind data are presented in Fig. 14, in which those stabilization diagrams of Acc 1, Acc 2, Acc 3, and Acc 4 caused stable poles at the frequency of $0.23 \mathrm{~Hz}$, which corresponds to the second mode. However, the second mode was not identified from blind data of Acc 5 and Acc 6. Moreover, for data Acc 5 and Acc 6, unstable 
poles are scattered widely in the low frequency band below $0.25 \mathrm{~Hz}$. The identified second mode shapes are presented in Fig. 15, which shows that the mode shapes obtained from Acc 1 and Acc 2 correspond well to the identified mode shapes from Sample 8, Sample 9, and Sample 10 presented in Fig. 5 and Sample 7 in Fig. 13. However, the lateral modes identified from Acc 3 and Acc 4 are more comparable to the modes identified from Sample 12 and Sample 13 rather than those identified from typhoon conditions. The torsional mode identified from the blind data of Acc 3 differs slightly from the modes of Acc 1, Acc 2, and Acc 4.

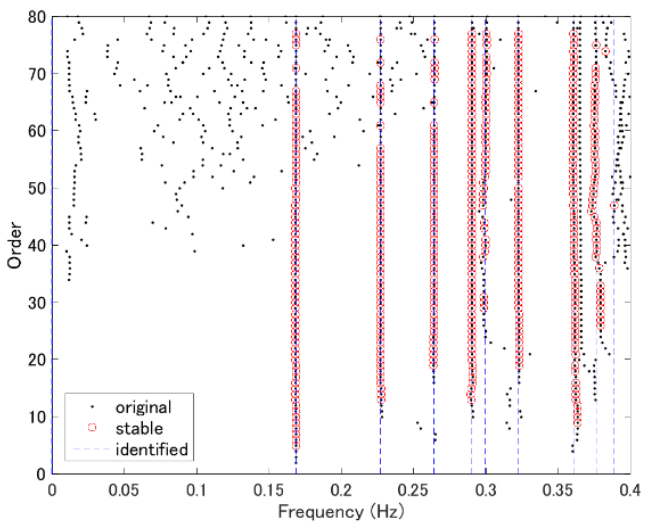

a) Acc 1

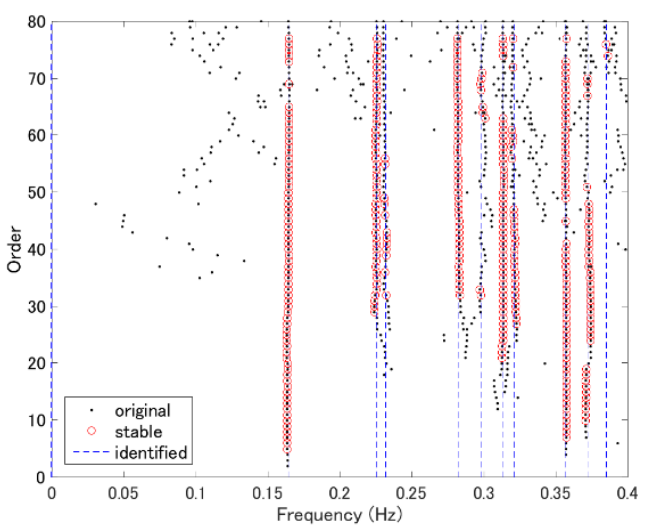

c) Acc 3

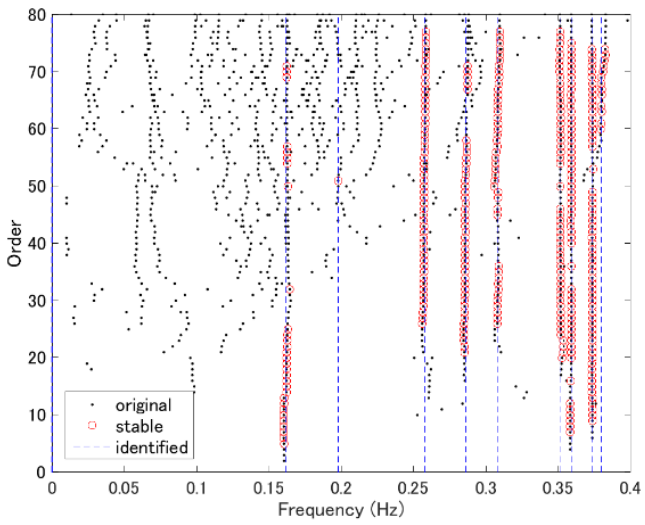

e) Acc 5

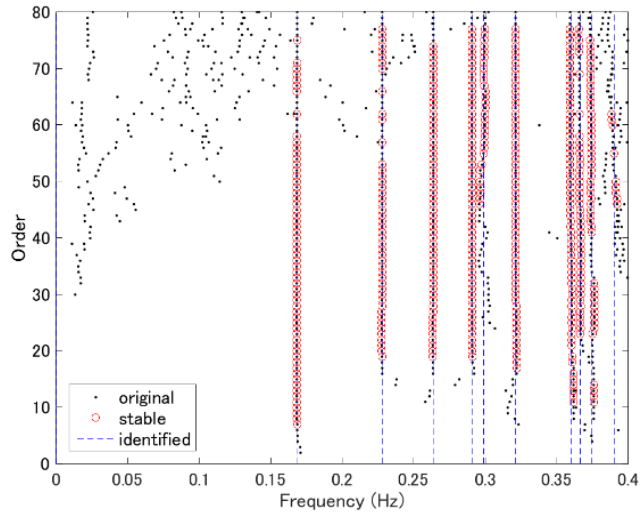

b) Acc 2

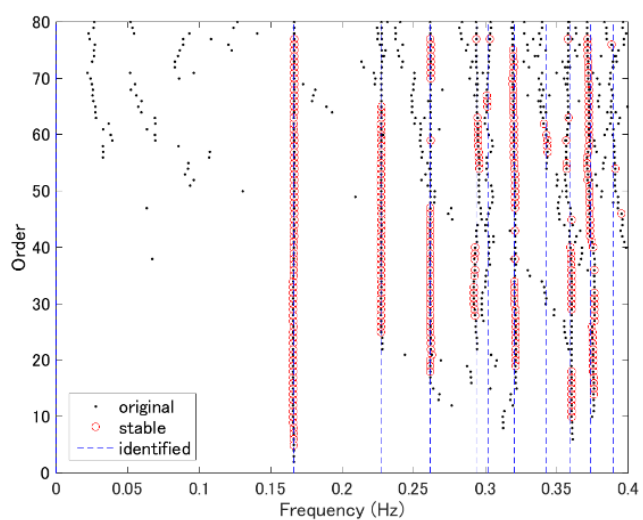

d) Acc 4

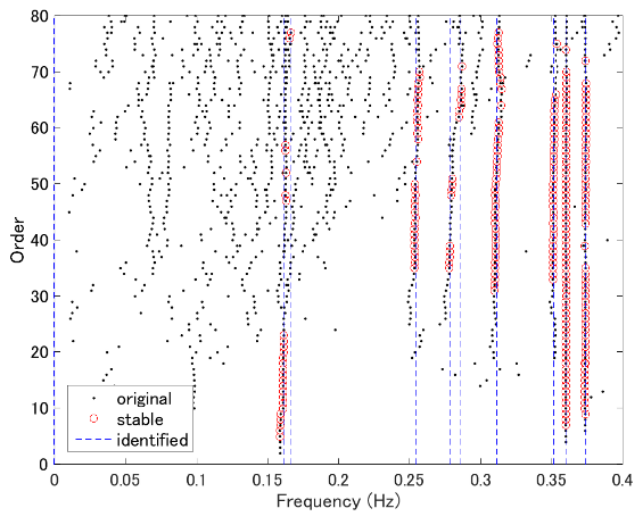

f) Acc 6

Fig. 14 Stabilization diagrams obtained from blind data. 


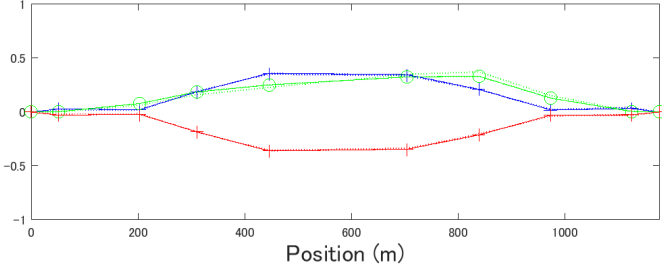

a) Acc 1

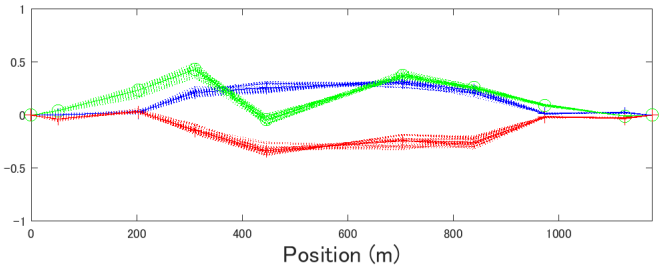

c) Acc 3

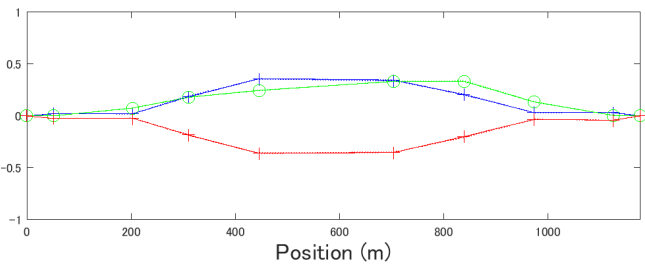

b) Acc 2

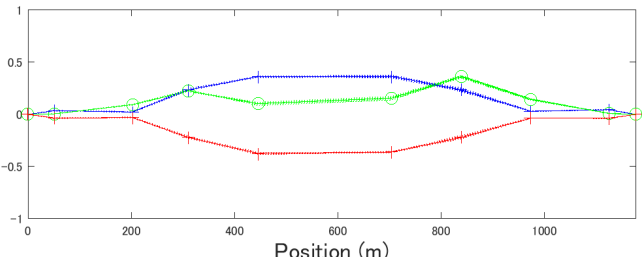

d) Acc 4

Fig. 15 Mode shape of the second mode obtained from blind data.

Observations suggest that data Acc 5 and Acc 6 can be categorized as data measured under weak wind conditions. In contrast, Acc 1, Acc 2, Acc 3, and Acc 4 can be categorized as data measured under strong wind conditions such as typhoon and critical wind conditions. More specifically, Acc1 and Acc 2 can be regarded as data measured under typhoon conditions because both torsional and lateral modes for the second mode correspond well to the mode of non-blind data obtained under typhoon conditions. However, it is natural to categorize Acc 3 and Acc 4 as data obtained under the critical wind conditions because the lateral mode was not comparable with that mode under typhoon conditions, as discussed already in Chapter 4.

The CCRs of the blind data are shown as presented in Fig. 16, which shows that for data from Acc 1 to Acc 4, the CCR reached 90\% with model order of 50 whereas Acc 5 and Acc 6 need model order of 80 . The observation from CCR also supports the result that the blind data Acc 1 to Acc 4 can be categorized as data obtained under strong wind conditions such as typhoon and critical wind conditions, whereas Acc 5 and Acc 6 can be categorized as data obtained under weak wind conditions.

Finally the PSD curves of the lateral acceleration of the blind data were investigated. Those PSD curves are presented in Fig. 17, which shows that PSD curves of Acc 1 - Acc 4 have dominant frequency at $0.23 \mathrm{~Hz}$ (second modes) as observed already from stabilization diagrams. However, for Acc 5 and Acc 6, the PSD at $0.23 \mathrm{~Hz}$ was not clear but the PSD in lower frequency range below $0.18 \mathrm{~Hz}$ were stronger than other data. Therefore, following the discussion on the non-blind data done in section 4.2, the data Acc 1 - Acc 4 are categorized as measured data obtained under strong wind conditions such as typhoon and critical wind conditions, whereas data Acc 5 and Acc 6 were regarded as measured data obtained under a weak wind condition. 


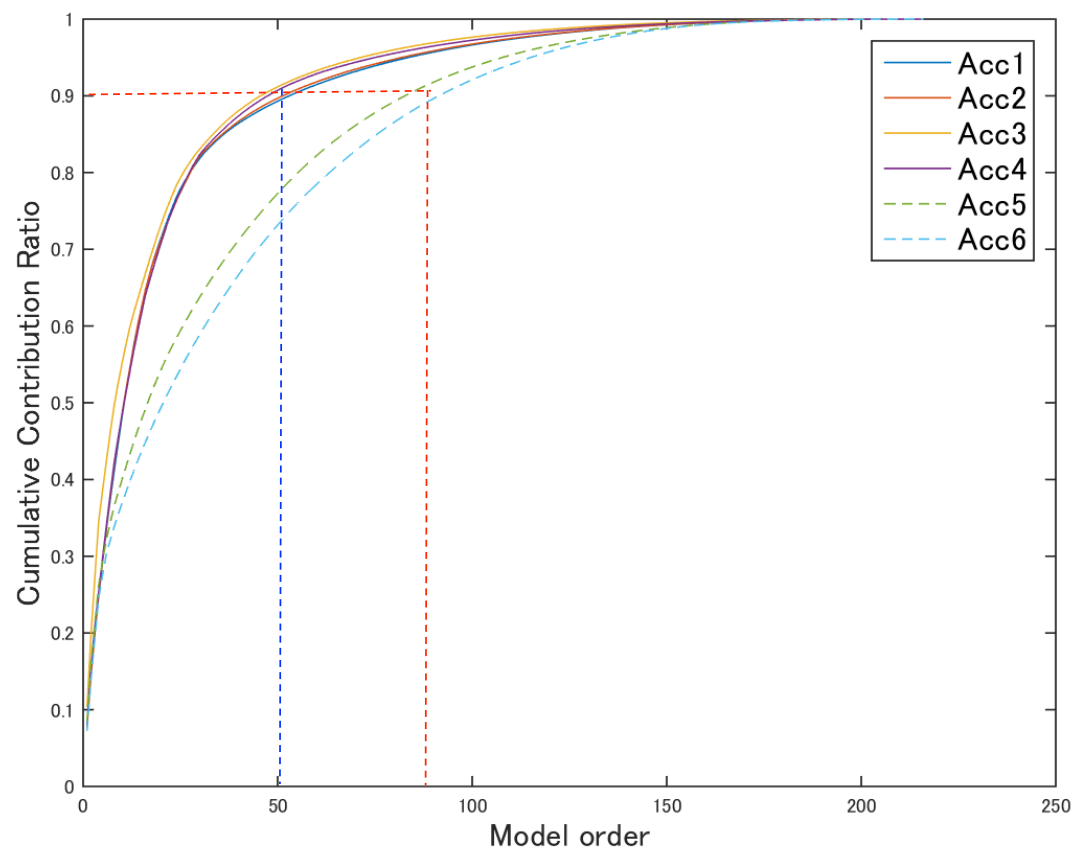

Fig. 16 Cumulative contribution ratios of singular values obtained from the blind data.

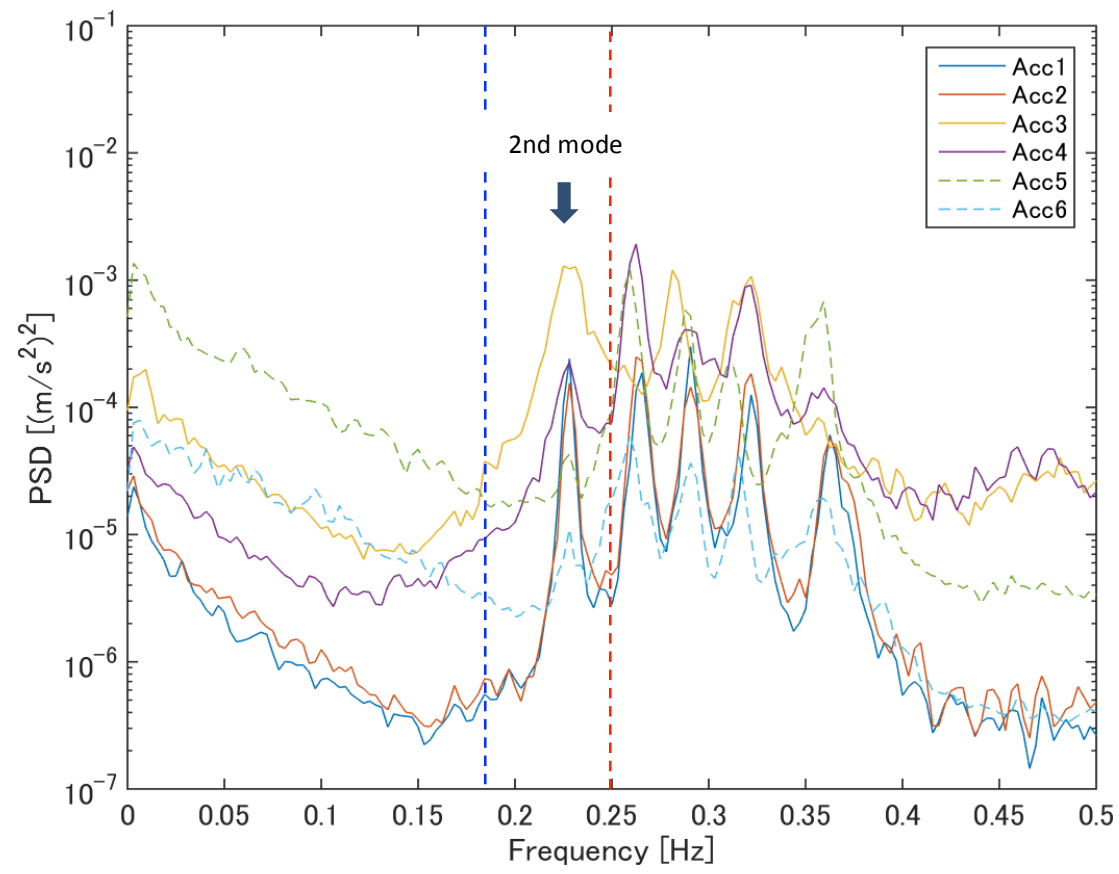

Fig. 17 Mean value of the lateral acceleration PSD obtained from the blind data.

\section{Concluding Remarks}

The mode identifiability of a multi-span cable-stayed bridge under different wind conditions was investigated as a benchmark study. Stochastic subspace identification (SSI) 
was applied to identify mode properties. Stabilization diagrams of given non-blind and blind data were examined to extract stable modes. The CCR estimated from singular values of the identified system model is adopted to investigate the contributions of poles of the estimated system models to the dynamical behavior of the structure under different wind conditions.

Identified modal properties from the non-blind data compared well with results of earlier studies: a deficient second mode was observed for which identifiability depends on wind conditions.

The stabilization diagrams of data measured under weak wind conditions showed widely scattered unstable poles in the frequency band below $0.23 \mathrm{~Hz}$, but no clear stable pole at the frequency of the second mode $(0.23 \mathrm{~Hz})$. However, under typhoon conditions, less unstable poles were observed in the frequency band below $0.23 \mathrm{~Hz}$; stable poles were observed on the frequency of the second mode. In other words, lower modes might be contaminated easily by unknown noise under weak wind conditions. However, under a typhoon, structural modes were well excited and the system matrix was dominated by structural information rather than noise, which might link with the mode identifiability of the second mode. In a stabilization diagram obtained from one sample under strong winds, unstable poles are distributed much more widely than under typhoon conditions and other critical wind conditions. This observation implies that those unstable poles do not depend merely on the mean hourly wind speed.

Observations from PSD curves of lateral acceleration showed that, especially in the low frequency band below $0.18 \mathrm{~Hz}$, most PSDs of the lateral accelerations under weak winds exceed the PSDs of the typhoon condition, even though the exciting wind speed is less than that under typhoon conditions. A possible reason for stronger PSDs appearing under the weaker wind conditions than those of a typhoon below the frequency range of $0.18 \mathrm{~Hz}$ might be the influence of traffic because traffic is controlled during the typhoon.

Observations also demonstrated that the CCR reaches a higher ratio with less model order under typhoon conditions in comparison to the conditions under weak wind. This observation implies that spurious poles observed in stabilization diagrams reduced the quantity of the information contained in the identified system models.

One sample under the typhoon condition failed to identify the second mode when all given data were used to calculate SSI. However, the second mode was identified when the steady part of the data was used for the calculation, which implies that the unsteadiness of the excitation is one cause of a failure to identify the mode shapes.

Blind data were also investigated using the stabilization diagram, CCR, and PSD curves. Observations suggest the following: 1) Blind data Acc 1 and Acc 2 were categorized as data obtained under a typhoon as both torsional and lateral modes for the second mode, corresponding well to the mode of non-blind data obtained under typhoon conditions. 2) 
Blind data Acc 3 and Acc 4 were categorized as data obtained under wind conditions with wind speeds around the critical value of $7.5 \mathrm{~m} / \mathrm{s}$ because the lateral mode was not comparable with that mode under typhoon conditions, even though the torsional mode data were comparable with that of non-blind data obtained under typhoon conditions. 3) Blind data Acc 5 and Acc 6 were categorized as data measured under weak wind conditions because the second mode was not identified. Moreover, unstable poles were scattered widely in the low frequency band below $0.25 \mathrm{~Hz}$.

Although this study did not fully resolve the questions of the benchmark study, the CCRs of singular values of the system model suggest the feasibility of their use to assess the performance of mode identification. The next step for this avenue of research is to investigate the influence of the traffic and cable vibrations on mode identifiability.

\section{Acknowledgments}

The authors would like to thank Prof. Y.Q. Ni of Hong Kong Polytechnic University for providing monitoring data and materials relevant to the Ting Kau Bridge and organizing the benchmark study. The authors thank Prof. H. Shirato of Kyoto University for his valuable comments related to cable-stayed bridges and cable vibrations.

\section{References}

Akaike, H. (1973), "Information theory and extension of the maximum likelihood principle", Proc. Second Int. Symp. on Information Theory, Petrov, B. N. and Csaki. F., Tsahkadsov, Armenia, USSR, pp. 267-281.

Bergermann, R. and Schlaich, M. (1996), “Ting Kau Bridge, Hong Kong”, Struct. Eng. Int., 6, (3), 152-154.

Brincker, R., Zhang, L. and Andersen, P. (2000), "Modal identification from ambient response using frequency domain decomposition", IMAC XVIII, San Antonio, USA.

Chang, K.C., Kim, C.W. and Kitauchi, S. (2013), "Stability diagram aided multivariate AR analysis for identifying the modal parameters of al steel truss bridge subjected to artificial damage", Proc. of the 13th East Asia-Pacific Conf. on Structural Eng. and Constr. (EASEC-13), September 11-13, Sapporo, Japan.

Daniels, R.W. (1974), “Approximation Methods for Electronic Filter Design”, McGraw-Hill, New York.

Deraemaeker, A., Reynders, E., De Roeck, G. and Kullaa, J. (2007), "Vibration-based structural health monitoring using output-only measurements under changing environment”, Mechanical Systems and Signal Processing, 22(1), 34-56. 
He, X. and De Roeck, G. (1997), "System identification of mechanical structures by a high-order multivariate autoregressive model, Computers and Structures, 64(1-4), 341-351.

Heylen, W., Lammens, S. and Sas, P. (1997), "Modal Analysis Theory and Testing”, K.U. Leuven, Belgium.

Kim, C.W. and Chang, K.C. (2014a), “A Field Experiment on a Simply Supported Steel Truss Bridge for Damage Detection Utilizing Statistical Patterns of Identified Modal Parameters", Life-Cycle of Structural Systems: Design, Assessment, Maintenance and Management, Furuta, Frangopol, Akiyama (Eds.), Proc. of the Fourth Int. Symp. On Life-Cycle Civil Eng., pp.361-368, Nov. 16-19, 2014, Tokyo, Japan.

Kim, C.W., Isemoto, R., Sugiura, K. and Kawatani, M. (2013), "Structural Fault Detection of Bridges based on Linear System Parameter and MTS Method", J. of JSCE, 1(1), 32-43.

Kim, C.W., Kitauchi, S., Sugiura, K., Kawatani, M. (2014b), "Utilizing reproduced autoregressive model for damage detection of real truss bridges", Life-Cycle of Structural Systems: Design, Assessment, Maintenance and Management, Furuta, Frangopol, Akiyama (Eds.), Proc. of the Fourth Int. Symp. On Life-Cycle Civil Eng., pp.369-376, Nov. 16-19, 2014, Tokyo, Japan.

Ko, J.M. and Ni, Y.Q. (2005), “Technology developments in structural health monitoring of large-scale bridges”, Eng. Struct., 27(12), 1715-1725.

Ni, Y.Q., Wang, Y.W. and Xia, Y.X. (2015), "Investigation of mode identifiability of a cable-stayed bridge: comparison from ambient vibration responses and from typhoon-induced dynamic responses", Smart Struct. Syst., 15(2), 447-468.

Peeters, B., Dammekens, F., Magalhaes, F., Van der Auweraer, H., and Cunha, A. (2006), "Multi-run Operational Modal analysis of the Guadiana Cable-stayed Bridge", Proc. of IMAC24, St. Louis, MO, January.

Van Overschee, P. and De Moor, B (1996), "Subspace Identification for Linear Systems", Kluwer Academic Publishers.

Wenzel, H. and Pichler, D. (2006), “Ambient Vibration Monitoring”, John Wiley \& Sons.

Wong, K.Y. (2004), "Instrumentation and health monitoring of cable-supported bridges", Struct. Control Health Monit., 11(2), 91-124.

Zhang, Q.W., (2007), "Statistical damage identification for bridges using ambient vibration data", Computers and Structures, 85(7-8), 476-485. 